\title{
Effect of ginseng polysaccharides on NK cell cytotoxicity in immunosuppressed mice
}

\author{
YAOYAO SUN ${ }^{1}$, MOFEI GUO ${ }^{1}$, YUANJIE FENG ${ }^{1}$, HUIFANG ZHENG $^{1}$, PING LEI $^{2}$, XIANDE MA $^{3}$, \\ XIAOWEI HAN ${ }^{2}$, HONGQUAN GUAN ${ }^{2}$ and DIANDONG HOU ${ }^{2}$ \\ ${ }^{1}$ The First Clinical Medical Institute, Liaoning University of Traditional Chinese Medicine; \\ ${ }^{2}$ Department of Immunization and Pathogen Biology, Basic Medical Science College, \\ Liaoning University of Traditional Chinese Medicine; \\ ${ }^{3}$ Experiment and Technology Center, Liaoning University of Traditional Chinese Medicine, \\ Shenyang, Liaoning 110032, P.R. China
}

Received March 24, 2015; Accepted April 26, 2016

DOI: $10.3892 /$ etm.2016.3840

\begin{abstract}
The aim of the present study was to investigate the effects of Ginseng polysaccharides (GPS) on natural killer (NK) cell cytotoxicity in immunosuppressed mice. Cyclophosphamide (Cy) was used to construct an immunosuppressed mouse model. The mice in each group were submitted to gavages with 200 or $400 \mathrm{mg} / \mathrm{kg}$ GPS every day for 10 days. Magnetic-activated cell sorting was used to isolate spleen NK cells, and the NK cell cytotoxicity, blood distribution, expression levels of perforin and granzyme, and the mRNA expression levels of interferon (IFN) $-\gamma$ were detected. Compared with the normal control group, the cytotoxicity and proportion of NK cells in the blood, and the expression levels of perforin, granzyme and IFN- $\gamma$ mRNA in the Cy model group were significantly reduced $(\mathrm{P}<0.05)$. In addition, compared with the Cy model group, the cytotoxicity and proportion of NK cells in the whole blood, and the expression levels of perforin and granzyme in the NK cells in the Cy + low-dose GPS and Cy + high-dose GPS groups were significantly increased $(\mathrm{P}<0.05)$. However, the mRNA expression levels of IFN- $\gamma$ in the NK cells did not significantly change ( $\mathrm{P}>0.05)$. Compared with the normal control group, the cytotoxicity and proportion of NK cells in the whole blood, and the expression levels of perforin in the $\mathrm{Cy}+$ low-dose GPS and the $\mathrm{Cy}+$ high-dose GPS groups were significantly lower $(\mathrm{P}<0.05)$.
\end{abstract}

Correspondence to: Dr Diandong Hou, Department of Immunization and Pathogen Biology, Basic Medical Science College, Liaoning University of Traditional Chinese Medicine, Chongshandonglu 79, Shenyang, Liaoning 110032, P.R. China E-mail: houdiandong@163.com

Abbreviations: GPS, ginseng polysaccharide; cy, cyclophosphamide; NK, natural killer; MACS, magnetic-activated cell sorting; FACS, fluorescence-activated cell sorting; IFN, interferon

Key words: ginseng polysaccharide, immunosuppression, natural killer cells, cytotoxicity
However, the expression levels of granzyme in the NK cells was not significantly different, as compared with the normal control group $(\mathrm{P}>0.05)$. These results suggested that GPS promotes NK cell cytotoxicity in immunosuppressed mice by increasing the number of NK cells in the whole blood and upregulating the expression of perforin and granzyme. Thus, the present study investigated the molecular mechanism underlying NK cell activation by GPS, the research showed that GPS have a wide application prospects in the treatment of cancer and immunodeficiency diseases.

\section{Introduction}

Ginseng is a traditional Chinese herbal medicine and is well-known as a medicinal plant throughout the world. Ginseng has been used for centuries for the treatment of numerous diseases, including cancer, hepatitis and immune deficiency diseases (1-3). To date, several components of Ginseng with broad medical functions have been identified (4-6). Ginseng polysaccharides (GPS) have been demonstrated to have immunomodulatory functions, such as the activation of macrophages and natural killer (NK) cells $(7,8)$. In China, GPS has become a commercial product for the treatment of various types of infections and cancer, as it is thought to increase host immune defense and immune surveillance $(9,10)$. Previous studies have reported that GPS may promote the cytotoxicity of NK cells in mice with neoplasms $(7,11)$. The clinical studies demonstrated that the activity of NK cells in the peripheral blood is markedly enhanced during the course of radiation therapy when combined with GPS. In addition, the side-effects caused by radiation therapy are reduced $(12,13)$. However, the mechanism underlying these effects remains to be elucidated.

The present study treated immunosuppressed mice with GPS and then observed the activity and distribution of NK cells in the blood prior to and following GPS treatment. In addition, the expression levels of perforin, granzyme and IFN- $\gamma$ mRNA in the NK cells were also detected. The aim of this study was to investigate the molecular mechanism underlying the NK cell activation by GPS, and to provide a basis for the study of the immunoregulatory activity of GPS. 


\section{Materials and methods}

Preparation of GPS solution. GPS (purity $>90 \%$ ) was purchased from Shaanxi Sinuote Biotech Co., Ltd. (Xi'an, China), and dissolved in phosphate-buffered saline (PBS).

Animals. Male BALB/c mice ( $\mathrm{n}=40$; weight, $18-22 \mathrm{~g}$; age, 4 weeks old) were obtained from Liaoning Chengda Biotechnology Co., Ltd. (Dalian, China). During the experimental period, the mice were housed in a room maintained under a $12 \mathrm{~h}$-light/dark cycle at $24^{\circ} \mathrm{C}$. Mice were provided with ad libitum access to standard laboratory pellet chow and fresh water.

The mice were randomly assigned to 4 groups with 10 mice/group as follows: Normal control group (A), cyclophosphamide (Cy) model group (B), Cy + low-dose GPS group (C), Cy + high-dose GPS group (D). The mice of groups $\mathrm{B}, \mathrm{C}$ and $\mathrm{D}$ were injected intraperitoneally with $50 \mathrm{mg} / \mathrm{kg}$ Cy (Shanxi Pude Pharmaceutical Co., Ltd., China) on days 1-3 once/day. On the 4th day, the $\mathrm{C}$ and $\mathrm{D}$ group mice were treated with 200 and $400 \mathrm{mg} / \mathrm{kg}$ GPS by gavage for 10 days, whereas the $\mathrm{A}$ and $\mathrm{B}$ group mice were given PBS by gavage. On the 14th day, the mice were sacrificed by cervical dislocation, and peripheral blood cells were obtained via heart puncture. In addition, the spleen was collected for analysis. All experimental procedures were conducted according to the guidelines provided by the ethical committee of experimental animal care at Liaoning University of Traditional Chinese Medicine (Shenyang, China).

NK cell preparation. Cells from the spleen were pooled and single-cell suspensions were prepared as follows: The spleen was thoroughly washed with ice-cold PBS to remove red blood cells and then immersed in PBS minced quickly, using a pair of sharp scissors to $\sim 1-\mathrm{mm}^{3}$ pieces. These were placed in a $40 \mu \mathrm{m}$ cell strainer containing spleen pieces into a $60 \mathrm{~mm}$ Petri dish and add a few milliliters of fresh medium. Using the barrel from a $2 \mathrm{ml}$ syringe, the spleens were pressed through the strainer. This was continued until only a small amount of fibrous tissue remained in the strainer. Using fresh medium and a Pasteur pipet, the Petri dish was rinsed, as was the cell strainer, to ensure that all cells have been recovered. Purified splenic NK cell populations were further isolated using magnetic-activated cell sorting (MACS) magnetic bead separation technology. Briefly, anti-NK cell DX5 MicroBeads (1:100) were used according to the manufacturer's protocol (Miltenyi Biotec $\mathrm{GmbH}$, Bergisch Gladbach, Germany) using the positive selection program 'PosselD' on the autoMACS Pro Separator (Miltenyi Biotec). The purity of the cells was routinely tested by fluorescence-activated cell sorting (FACS) and ranged from $87-91 \%$.

Quantification of NK cell cytotoxicity. The isolated NK cells $\left(1 \times 10^{5}\right.$ cells) were placed in each well of a 96-well plate in $50 \mu 1$ RPMI-1640 medium (Gibco; Thermo Fisher Scientific, Inc., Waltham, MA, USA), and then co-cultured with K562 (target) cells (The Type Culture Collection of the Chinese Academy of Sciences, Shanghai, China) at an effector-to-target ratio of 4:1 for $4 \mathrm{~h}$ at $37^{\circ} \mathrm{C}$.

The plates were centrifuged at speed $1500 \mathrm{x} g$ at $4^{\circ} \mathrm{C}$ for $5 \mathrm{~min}$, and $100 \mu \mathrm{l}$ supernatant was transferred to new 96-well plates. Subsequently, a lactate dehydrogenase-substrate mixture (100 $\mu 1$; cat. no. 11644793001; Roche Diagnostics, Risch-Rotkreuz, Switzerland) containing tetrazolium salt INT was added to the supernatants. Following incubation for $30 \mathrm{~min}$ in the dark at room temperature, the absorbance was measured using a Multiskan MK3 (Thermo Fisher Scientific, Inc.) at a wavelength of $492 \mathrm{~nm}$ against a reference wavelength of $600 \mathrm{~nm}$. Lysis percentage was calculated using the following equation:

$$
\text { Specific lysis (\%) }=\frac{O D_{\text {experiment }}-O D_{\text {spontaneous }}}{O D_{\text {maximun }}-O D_{\text {spontaneous }}} \times 100 \%
$$

Western blot analysis. The cells were washed three times with PBS and then lysed in radioimmunoprecipitation assay buffer in the presence of proteinase inhibitor cocktail (Sigma-Aldrich, St. Louis., MO, USA; and was formulated as follows: $150 \mathrm{mM} \mathrm{NaCl}, 1.0 \%$ IGEPAL CA-630, $0.5 \%$ sodium deoxycholate, $0.1 \%$ SDS, and $50 \mathrm{mM}$ Tris, $\mathrm{pH}$ 8.0). The protein concentration was determined using a bicinchoninic acid assay (Beijing Biosynthesis Biotechnology Co., Ltd., Beijing, China). Aliquots $(25 \mathrm{mg}$ ) were separated by $10 \%$ SDS-PAGE and transferred to nitrocellulose membranes. The membranes were probed with primary antibodies (Abcam, Cambridge, UK, dilution: 1:1,000) targeting perforin (cat. no. ab180773) and granzyme (cat. no. ab53097) at $4^{\circ} \mathrm{C}$ for $12 \mathrm{~h}$, washed extensively with $0.1 \%$ Tween-20 in PBS, and incubated with secondary antibody conjugated to horseradish peroxidase (1:10,000; cat. no. SA00001-2; Proteintech Group, Inc. Wuhan, China) and secondary antibody fluorescein isothiocyanate-conjugated DX5 (cat. no. MA5-16648; dilution, 1:100; Pierce Biotechnology; Thermo Fisher Scientific, Inc.) at room temperature for $1 \mathrm{~h}$. The intensity of the protein fragments was visualized using an X-ray image film processor (Kodak, Rochester, NY, USA). Bands from experiments were quantified by densitometry using Image $\mathbf{J}$ software (imagej.nih.gov/ij/), and results were normalized to Actin expression in each sample .

Flow cytometric analysis. Peripheral blood $(100 \mu \mathrm{l})$ was collected into ethylenediaminetetraacetic acid-coated tubes. Red blood cells were first lysed with FACS lysing solution (Beyotime Institute of Biotechnology, Jiangsu, China) and then washed twice with PBS containing $2 \%$ fetal bovine serum. The leukocytes were then incubated with fluorescein isothiocyanate-conjugated anti-mouse DX5 (CD49b; Pierce Biotechnology; Thermo Fisher Scientific, Inc.) for $30 \mathrm{~min}$ at room temperature. A FACScan flow cytometer (BD Biosciences, San Jose, CA, USA) was used and the data were analyzed using FlowJo software (FlowJoV10, Flexera Software Inc., Itasca, IL, USA).

Reverse transcription-quantitative polymerase chain reaction $(R T-q P C R)$. RT-qPCR was performed using SYBR-Green PCR Master-Mix (Applied Biosystems, Foster City, CA, USA) according to the manufacturer's protocol. Total RNA was extracted from the isolated NK cells using TRIzol reagent (Invitrogen; Thermo Fisher Scientific, Inc.). The purified total RNA was quantified by a Nano-Drop ND 1000 spectrophotometer (Nano-Drop Technologies, Wilmington, DE, USA). The fluorescent signals were detected using an ABI7700 Real-Time Sequence-Detection system (Applied Biosystems). 
The PCR conditions were as follows: Initial denaturation at $95^{\circ} \mathrm{C}$ for $5 \mathrm{~min}$ followed by 40 cycles of $\mathrm{PCR}$ reaction; denaturation at $95^{\circ} \mathrm{C}$ for $20 \mathrm{sec}$; annealing at $58^{\circ} \mathrm{C}$ for $30 \mathrm{sec}$ and elongation at $72^{\circ} \mathrm{C}$ for $30 \mathrm{sec}$. qPCR amplification was performed with $10 \mu \mathrm{l}$ final reaction mixture consisting of $0.5 \mu \mathrm{l}$ of reverse transcription reaction mixture, $0.1 \mu \mathrm{M}$ of each sense and antisense primer and 1x PCR mixture of SYBR-Green PCR Master-Mix (Applied Biosystems; Thermo Fisher Scientific, Inc.). The primers (listed in Table I) were synthesized by Invitrogen. All tests were run in triplicate. The values were normalized to $G A P D H$. Each group consisted of 3 parallel wells. Negative control (DNase/RNase-Free Water, Beijing Solarbio Science \& Technology Co., Ltd., Beijing, China). There was no RT control. The $2^{-\Delta \Delta \mathrm{Cq}}$ method was used to calculate relative expression quantify. (14)

Statistical analysis. The results are presented as means \pm standard deviation. A one-way analysis of variance (ANOVA) was used to compare multiple quantitative variables, followed by Student's t-test. $\mathrm{P}<0.05$ was considered to indicate a statistically significant result. SPSS statistical software (SPSS, Inc., Chicago, IL, USA) was used for statistical analyses.

\section{Results}

Effects of GPS on NK cell cytotoxicity in immunosuppressed mice. The effects of GPS on NK cell cytotoxicity in immunosuppressed mice are shown in Fig. 1. NK cell cytotoxicity in the Cy model group mice was significantly lower compared with that of the normal control group $(\mathrm{P}<0.01)$. Compared with the $\mathrm{Cy}$ model group, NK cell cytotoxicity in the $\mathrm{Cy}+$ low-dose GPS group was significantly increased $(\mathrm{P}<0.05)$; similar results were observed in the $\mathrm{Cy}+$ high-dose GPS group $(\mathrm{P}<0.01)$. Compared with the normal control group, NK cell cytotoxicity in the Cy + low-dose GPS group was significantly reduced $(\mathrm{P}<0.05)$, although no significant difference was observed between the $\mathrm{Cy}+$ high-dose GPS group and the normal control group ( $\mathrm{P}>0.05)$. Results showed that GPS could increase NK cell cytotoxicity in immunosuppressed mice.

Effects of GPS on NK cell distribution in the whole blood of immunosuppressed mice. The effects of GPS on NK cell distribution in the whole blood of immunosuppressed mice are shown in Fig. 2. The proportion of NK cells in the whole blood of the Cy model group mice was significantly lower compared with the normal control group $(\mathrm{P}<0.01)$. Compared with the $\mathrm{Cy}$ model group, the NK cell proportion in the whole blood of the $\mathrm{Cy}+$ low-dose GPS mice was significantly increased $(\mathrm{P}<0.05)$, ; similar results were observed in the $\mathrm{Cy}+$ high-dose GPS group $(\mathrm{P}<0.01)$. Compared with the normal control group, the proportion of $\mathrm{NK}$ cells in the whole blood of the Cy + low-dose GPS group was significantly reduced $(\mathrm{P}<0.01)$, and similar results were observed for the Cy + high-dose GPS group $(\mathrm{P}<0.05)$. Results showed that GPS could increase NK cell quantity in immunosuppressed mice.

Effect of GPS on the expression levels of perforin in the NK cells of immunosuppressed mice. The effects of GPS on the expression levels of perforin in the NK cells of
Table I. Primer sequences.

\begin{tabular}{ll}
\hline Gene & \multicolumn{1}{c}{ Primer sequences } \\
\hline IFN- $\gamma$ & Forward, 5'-CCTTTGGACCCTCTGACTT-3' \\
& Reverse, 5'-GGACCTGTGGGTTGTTGAC-3' \\
GAPDH & Forward, 5'-CCTCAAGATCATCAGCAAT-3' \\
& Reverse, 5'-CCATCCACAGTCTTCTGGGT-3'
\end{tabular}

IFN- $\gamma, \quad$ interferon- $\gamma ; \quad G A P D H, \quad$ glyceraldehyde 3 -phosphate dehydrogenase.

immunosuppressed mice are shown in Fig. 3. Perforin expression levels in the $\mathrm{Cy}$ model group were significantly downregulated compared with those in the normal control group $(\mathrm{P}<0.01)$. Compared with the $\mathrm{Cy}$ model group, perforin expression levels in the $\mathrm{Cy}+$ low-dose GPS group and the $\mathrm{Cy}+$ high-dose GPS group were significantly upregulated $(\mathrm{P}<0.05)$. However, the expression levels of perforin in the NK cells of the Cy + low-dose GPS and Cy + high-dose GPS groups were significantly lower compared with the normal control group $(\mathrm{P}<0.05)$. Results showed that GPS was able to increase expression levels of perforin of NK cells in immunosuppressed mice.

Effect of GPS on the expression levels of granzyme $B$ in the NK cells of immunosuppressed mice. The effects of GPS on the expression levels of granzyme B in the NK cells of immunosuppressed mice are showed in Fig. 4. Granzyme B expression levels in the NK cells of the $\mathrm{Cy}$ model group were significantly downregulated compared with those in the normal control grope $(\mathrm{P}<0.05)$. Compared with the $\mathrm{Cy}$ model group, granzyme B expression levels in the NK cells of $\mathrm{Cy}+$ low-dose and high-dose GPS groups was significantly upregulated $(\mathrm{P}<0.05)$. Compared with the normal control group however, the expression levels of granzyme B in the NK cells of the $\mathrm{Cy}+$ low-dose and high-dose GPS mice did not show any significant difference $(\mathrm{P}>0.05)$. Results indicated that GPS could increase expression levels of granzyme B in the NK cells in immunosuppressed mice.

Effect of GPS on the mRNA expression levels of IFN- $\gamma$ in the NK cells of immunosuppressed mice. The effects of GPS on the mRNA expression levels of IFN- $\gamma$ in the NK cells of immunosuppressed mice are shown in Fig. 5. Compared with the normal control group, the mRNA expression levels of IFN- $\gamma$ in the NK cells of the Cy model group mice were significantly downregulated $(\mathrm{P}<0.01)$. The mRNA expression levels of IFN- $\gamma$ in the NK cells of both the Cy + low-dose GPS group $(\mathrm{P}<0.01)$ and the $\mathrm{Cy}+$ high-dose GPS group $(\mathrm{P}<0.05)$ were also significantly downregulated compared with the normal control group. Compared with the Cy model group, the mRNA expression levels of IFN- $\gamma$ in the NK cells of both the Cy + low-dose GPS group and the $\mathrm{Cy}+$ high-dose GPS group showed no significant difference $(\mathrm{P}>0.05)$. Results indicated that the effects of GPS on mRNA expression levels of IFN- $\gamma$ of the NK cells in immunosuppressed mice were not significant. 


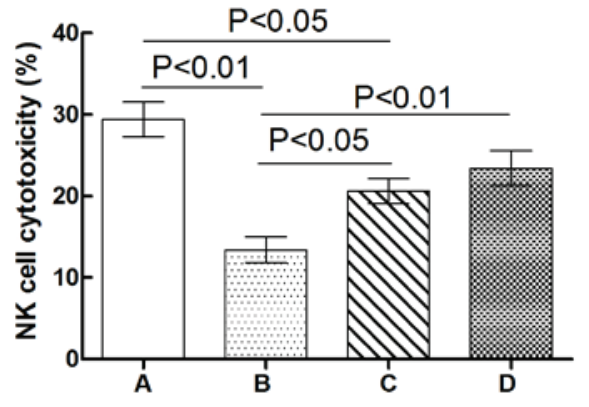

Figure 1. Effects of GPS on NK cell cytotoxicity in immunosuppressed mice. A, Normal control group; B, Cy model group; C, Cy + low-dose GPS group; $\mathrm{D}, \mathrm{Cy}+$ high-dose GPS group. NK, natural killer; $\mathrm{Cy}$, cyclophosphamide; GPS, Ginseng polysaccharides.
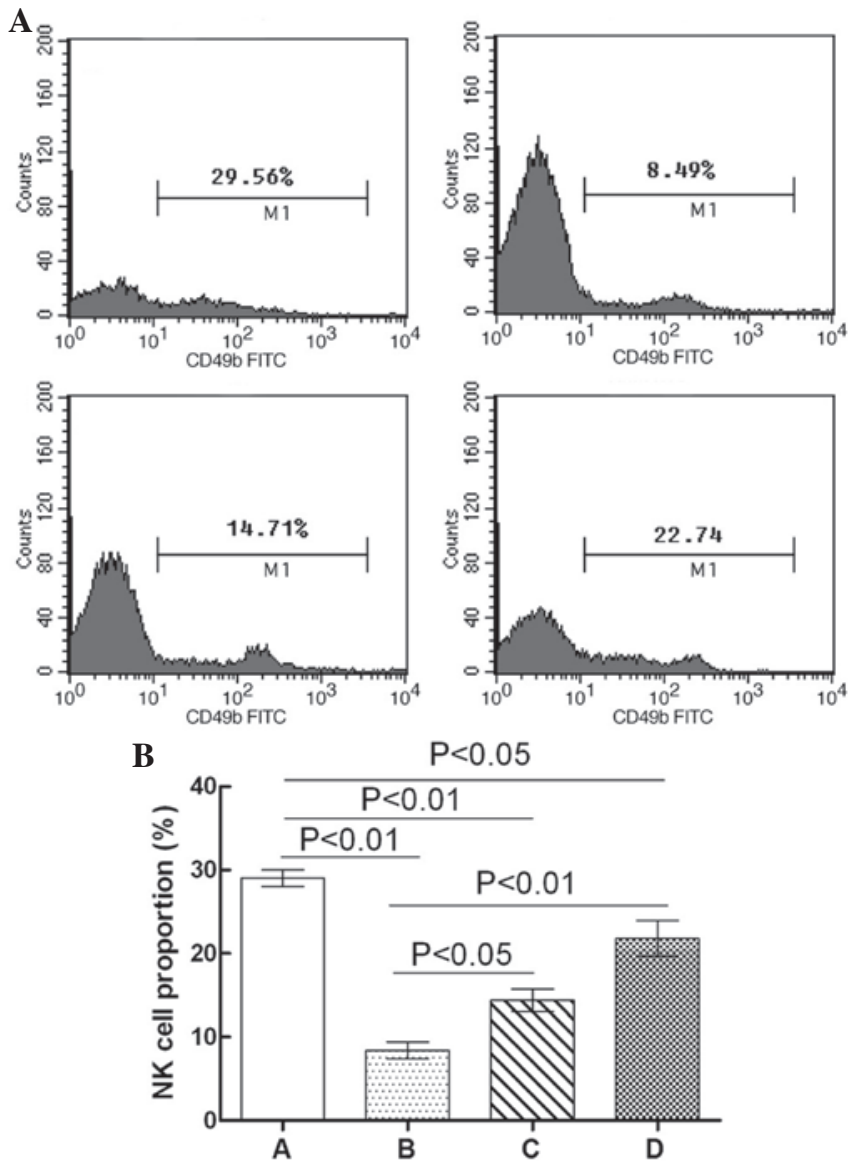

Figure 2. Effect of GPS on the distribution of NK cells in whole blood of immunosuppressed mice. (A) Flow cytometry was performed to analyze CD49b expression in NK cells. (B) Statistical analysis of NK cell proportion. A, Normal control group; B, Cy model group; $\mathrm{C}, \mathrm{Cy}+$ low-dose GPS group; $\mathrm{D}, \mathrm{Cy}+$ high-dose GPS group. NK, natural killer; GPS, Ginseng polysaccharides.

\section{Discussion}

NK cells are effector cells of the innate immune system and act as the first line of defense against tumors or viral infection $(15,16)$. While killing target cells, NK cells do not have to be sensitized by antigens and have no major histocompatibility complex restriction (17). NK cells are able to cause the lysis and apoptosis of target cells by releasing perforins and
A

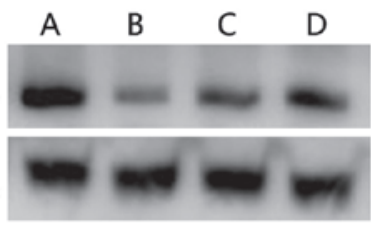

B

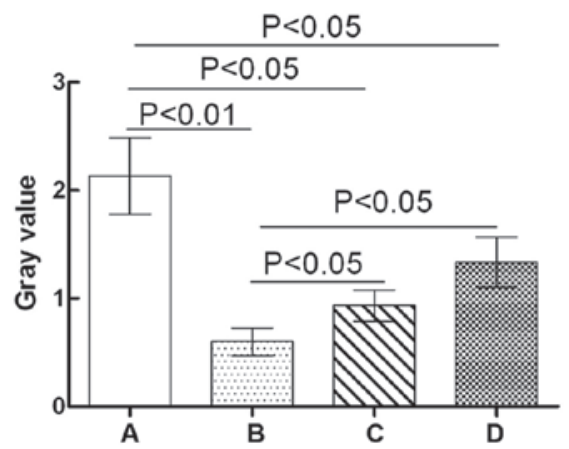

Figure 3. Effect of GPS on perforin expression levels in NK cells in immunosuppressed mice. (A) Western blot analysis of perforin expression levels. (B) Bands from three experiments of western blot were quantified by densitometry, and results were normalized to actin expression in each sample, and plotted with the standard error. A, Normal control group; B, Cy model group; $\mathrm{C}, \mathrm{Cy}+$ low-dose GPS group; D, Cy + high-dose GPS group. NK, natural killer; GPS, Ginseng polysaccharides.

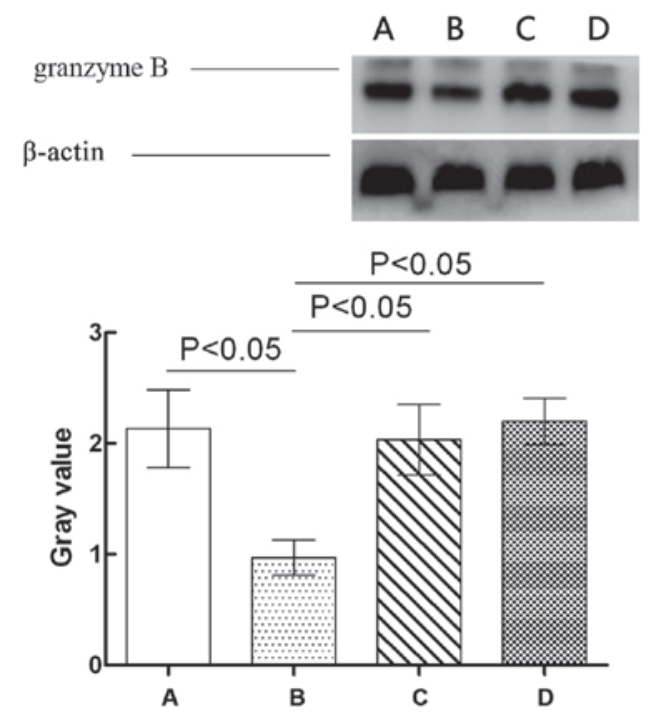

Figure 4. Effect of GPS on the expression levels of granzyme B in the NK cells of immunosuppressed mice. A, Normal control group; B, Cy model group; C, Cy + low-dose GPS group; $\mathrm{D}, \mathrm{Cy}+$ high-dose GPS group. NK, natural killer; GPS, Ginseng polysaccharides.

granzymes $(18,19)$. In addition, NK cells exhibit anti-tumor and anti-viral activity by releasing various types of cytokines, such as IFN- $\gamma(20-22)$. Investigators have demonstrated that GPS promotes NK cell cytotoxicity and anti-tumor activity, but few studies have investigated the mechanisms underlying these effects $(23,24)$.

In the present study, compared with the normal control group, the cytotoxicity and proportion of NK cells in the blood, and the expressing levels of perforin, granzyme and IFN- $\gamma$ mRNA in the immunosuppressed model group were significantly reduced, demonstrating that the immunosuppressed 


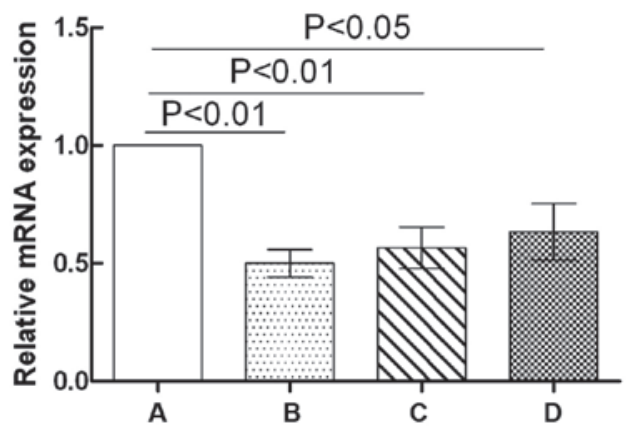

Figure 5. Effect of GPS on the mRNA expression levels of IFN- $\gamma$ in the NK cells of immunosuppressed mice. A, Normal control group; B, Cy model group; C, Cy + low-dose GPS group; D, Cy + high-dose GPS group. NK, natural killer; GPS, Ginseng polysaccharides; IFN- $\gamma$, interferon- $\gamma$.

mouse model was successfully created. Compared with the Cy model group, the cytotoxicity and proportion of NK cells in the blood of both the Cy + low-dose GPS group and the $\mathrm{Cy}+$ high-dose GPS group were significantly increased, suggesting that GPS was able to improve NK cell cytotoxicity in the immunosuppressed mice and promote NK cells differentiation. Compared with the $\mathrm{Cy}$ model group, the expression levels of perforin and granzyme in both the $\mathrm{Cy}+$ low-dose GPS group and the $\mathrm{Cy}+$ high-dose GPS group were significantly increased. This increase in expression levels was dependent on the dosage of GPS, and was higher in the high-dose GPS group. These results suggested that the mechanism underlying the increase in NK cell cytotoxicity induced by GPS may be associated with the ability of GPS to promote the expression and release of perforins and granzymes. Furthermore, compared with the $\mathrm{Cy}$ model group, the mRNA expression levels of IFN- $\gamma$ in the NK cells of the $\mathrm{Cy}+$ low-dose GPS group and the $\mathrm{Cy}+$ high-dose GPS group showed no significant difference, indicating that the effects of GPS on the expression levels of IFN- $\gamma$ mRNA in immunosuppressive mice were not obvious. However, the effect of GPS on the levels of IFN- $\gamma$ protein expression require further study.

In conclusion, the present study demonstrated that GPS is able to promote NK cell cytotoxicity in immunosuppressed mice by increasing the proportion of NK cells in the blood and by inducing the increased expression of perforins and granzymes. Thus, the present study investigated the molecular mechanism underlying the activation of NK cells activation GPS, and indicated that GPS has a wide application in the treatment of cancer and immunodeficiency diseases.

\section{Acknowledgements}

The present study was supported by the National Natural Science Foundation of China (grant no. 81303079).

\section{References}

1. Kwak JH, Park JY, Lee D, Kwak JY, Park EH, Kim KH, Park HJ, Kim HY, Jang HJ, Ham J, et al: Inhibitory effects of ginseng sapogenins on the proliferation of triple negative breast cancer MDA-MB-231 cells. Bioorg Med Chem Lett 24: 5409-5412, 2014.
2. Lee $\mathrm{MH}$, Lee $\mathrm{BH}$, Lee $\mathrm{S}$ and Choi C: Reduction of hepatitis $\mathrm{A}$ virus on FRhK-4 cells treated with Korean red ginseng extract and ginsenosides. J Food Sci 78: M1412-M1415, 2013.

3. Jiao L, Zhang X, Li B, Liu Z, Wang M and Liu S: Anti-tumour and immunomodulatory activities of oligosaccharides isolated from Panax ginseng C.A. Meyer. Int J Biol Macromol 65: 229-233, 2014.

4. Li C, Cai J, Geng J, Li Y, Wang Z and Li R: Purification, characterization and anticancer activity of a polysaccharide from Panax ginseng. Int J Biol Macromol 51: 968-973, 2012.

5. Ban JY, Kang SW, Lee JS, Chung JH, Ko YG and Choi HS: Korean red ginseng protects against neuronal damage induced by transient focal ischemia in rats. Exp Ther Med 3: 693-698, 2012.

6. Yang JW and Kim SS: Ginsenoside Rc promotes anti-adipogenic activity on 3T3-L1 adipocytes by down-regulating $\mathrm{C} / \mathrm{EBP} \alpha$ and PPAR $\gamma$. Molecules 20: 1293-1303, 2015.

7. Ni W, Zhang X, Wang B, Chen Y, Han H, Fan Y, Zhou Y and Tai G: Antitumor activities and immunomodulatory effects of ginseng neutral polysaccharides in combination with 5-fluorouracil. J Med Food 13: 270-277, 2010.

8. Wang J, Zuo G, Li J, Guan T, Li C, Jiang R, Xie B, Lin X, Li F, Wang Y and Chen D: Induction of tumoricidal activity in mouse peritoneal macrophages by ginseng polysaccharide. Int J Biol Macromol 46: 389-395, 2010.

9. Cai JP, Wu YJ, Li C, Feng MY, Shi QT, Li R, Wang ZY and Geng JS: Panax ginseng polysaccharide suppresses metastasis via modulating Twist expression in gastriccancer. Int $\mathbf{J}$ Biol Macromol 57: 22-25, 2013.

10. Cheng H, Li S, Fan Y, Gao X, Hao M, Wang J, Zhang X, Tai G and Zhou Y: Comparative studies of the antiproliferative effects of ginseng polysaccharides on HT-29 human colon cancer cells. Med Oncol 28: 175-181, 2011.

11. Yamaoka Y, Kawakita T, Kaneko M and Nomoto K: A polysaccharide fraction of Zizyphi fructus in augmenting natural killer activity by oral administration. Biol Pharm Bull 19: 936-939, 1996.

12. Xie FY, Zeng ZF and Huang HY: Clinical observation on nasopharyngeal carcinoma treated with combined therapy of radiotherapy and ginseng polysaccharide injection. Zhongguo Zhong Xi Yi Jie He Za Zhi 21: 332-334, 2001 (In Chinese).

13. Cho YJ, Son HJ and Kim KS: A 14-week randomized, placebo-controlled, double-blind clinical trial to evaluate the efficacy and safety of ginseng polysaccharide (Y-75). J Transl Med 12: 283, 2014.

14. Livak KJ and Schmittgen TD: Analysis of relative gene expression data using real-time quantitative PCR and the $2-\Delta \Delta \mathrm{Ct}$ method. Methods 25: 402-408, 2001.

15. Watzl C: How to trigger a killer: Modulation of natural killer cell reactivity on many levels. Adv Immunol 124: 137-170, 2014.

16. Eddy JL, Krukowski K, Janusek L and Mathews HL: Glucocorticoids regulate natural killer cell function epigenetically. Cell Immunol 290: 120-130, 2014.

17. Cox JH: Evaluation of natural killer cell activity. Methods Mol Med 17: 383-389, 1999.

18. Jiang W, Zhang C, Tian Z and Zhang J: hIFN- $\alpha$ gene modification augments human natural killer cell line anti-human hepatocellular carcinoma function. Gene Ther 20: 1062-1069, 2013.

19. Li Q, Kobayashi M, Wakayama Y, Inagaki H, Katsumata M, Hirata Y, Hirata K, Shimizu T, Kawada T, Park BJ, et al: Effect of phytoncide from trees on human natural killer cell function. Int J Immunopathol Pharmacol 22: 951-959, 2009.

20. Ahlenstiel G: The natural killer cell response to HCV infection. Immune Netw 13: 168-176, 2013.

21. Campbell KS and Hasegawa J: Natural killer cell biology: An update and future directions. J Allergy Clin Immunol 132: 536-544, 2013.

22. Lin SJ, Yan DC, Lee WI, Kuo ML, Hsiao HS and Lee PY: Effect of azithromycin on natural killer cell function. Int Immunopharmacol 13: 8-14, 2012.

23. Zhou X, Shi H, Jiang G, Zhou Y and Xu J: Antitumor activities of ginseng polysaccharide in C57BL/6 mice with Lewis lung carcinoma. Tumour Biol 35: 12561-12566, 2014.

24. Jiao L, Zhang X, Li B, Liu Z, Wang M and Liu S: Anti-tumour and immunomodulatory activities of oligosaccharides isolated from Panax ginseng C.A. Meyer. Int J Biol Macromol 65: 229-233, 2014. 